\title{
Corps de l'écrivain et érotisme littéraire chez Claude Simon
}

\section{Paul Dirkx}

\section{(2) OpenEdition}

1 Journals

Édition électronique

URL : http://journals.openedition.org/ccs/1059

DOI : $10.4000 /$ ccs. 1059

ISSN : 2558-782X

Éditeur :

Presses universitaires de Rennes, Association des lecteurs de Claude Simon

\section{Édition imprimée}

Date de publication : 1 décembre 2017

Pagination : 145-160

ISBN : 978-2-7535-5482-5

ISSN : $1774-9425$

\section{Référence électronique}

Paul Dirkx, "Corps de l'écrivain et érotisme littéraire chez Claude Simon », Cahiers Claude Simon [En ligne], 12 | 2017, mis en ligne le 07 décembre 2018, consulté le 13 février 2020. URL : http://

journals.openedition.org/ccs/1059; DOI : 10.4000/ccs.1059 


\title{
CORPS DE L'ÉCRIVAIN ET ÉROTISME LITTÉRAIRE CHEZ CLAUDE SIMON
}

\author{
Paul DIRKX \\ Université de Lorraine
}

Entre Triptyque et Le Jardin des Plantes $^{1}$, vingt-quatre ans se sont écoulés pendant lesquels l'écriture de Claude Simon a connu d'importantes évolutions. On en identifiera ici quelques-unes, en prêtant attention à tout ce qui a trait au corps dans ces deux romans. Le corps apparaît en effet comme un analyseur méconnu de la poétique simonienne et de ses mutations au fil de l'ouvre ${ }^{2}$.

Les romans de Claude Simon se distinguent ainsi par une narration dont certains traits sont directement liés aux corps ${ }^{3}$. Les diégèses s'avèrent saturées de corporéité, d'autant plus que le narrateur simonien a tendance à fondre les sujets et les objets dans une même catégorie, celle des corps d'une manière générale. Au sein de cette catégorie, les différences entre corps inanimés et corps animés (humains ou non) s'effacent volontiers devant leur commune soumission aux forces primitives et antagoniques qui gouvernent le monde: la vie et la mort, le mouvement et l'inertie, l'ombre et la lumière, etc. La narration se résume souvent à l'observation des effets que ces couples antinomiques ne cessent de produire sur les corps, lesquels s'en trouvent constamment modifiés, pour l'essentiel animés ou à l'inverse immobilisés

1. Minuit, 1973 et 1997. Dans ce qui suit, les références aux œuvres de Claude Simon se feront par la mention de la page dans l'édition de "La Pléiade " (2006 et 2013).

2. Voir P. Dirkx, "Claude Simon: antinomie et corps écrivant ", P. Dirkx et P. Mougin (dir.), Claude Simon: situations, Lyon, ENS Éd., 2011, p. 179-197.

3. Pour une analyse du caractère sensitif du style simonien, voir D. Zemmour, Une syntaxe du sensible. Claude Simon et l'écriture de la perception, PU de Paris-Sorbonne, 2008. 
de l'intérieur ou de l'extérieur - on en trouvera plus d'un exemple dans ce qui suit. Cette sensibilité aux corps dans leur diversité et dans leur instabilité s'affirme dès le début de l'œuvre et s'aiguise d'un roman à l'autre. Elle semble toujours moins concernée par ce qui arrive aux corps que par leurs rapports mutuels. C'est du moins l'hypothèse qui sera mise à l'épreuve à travers une lecture de Triptyque, puis du Jardin des Plantes.

\section{TRIPTYQUE OU «LE DÉSIR D’ÉCRIRE ${ }^{4}$ »}

De l'intérêt croissant porté aux corps comme matériau poétique, le roman Triptyque offre une illustration quasi programmatique. La narration-observation y est le fait d'une instance aux apparences impersonnelles et insaisissables, qui parfois semble déléguer ses prérogatives à une "caméra » toute matérielle qui rend son statut encore plus flou. Ce regard clinique tantôt dissèque les corps, tantôt les fait entrer en fusion, tantôt les deux à la fois, suivant des logiques compositionnelles qui se trouvent régulièrement explicitées, comme mises en abyme, dans des passages où le narrateur-observateur prend alors des allures de critique à la fois littéraire, pictural et cinématographique ${ }^{5}$. Le ton général du roman est d'ailleurs celui d'un commentaire analytique de phénomènes complexes qui, pour issus de la réalité qu'ils soient, semblent participer d'un gigantesque objet d'art sophistiqué ${ }^{\text {. }}$

La composition doit beaucoup aux couples antinomiques évoqués en préambule. Le couple "mobilité »/«immobilité » y joue un rôle moteur, non seulement par son omniprésence, mais aussi du fait que le lexème «immobil-»-78 occurrences, soit une toutes les 2,8 pages - est d'autant plus signifiant que "mobil-» ne figure pas une seule fois dans le texte ${ }^{7}$. Le caractère antinomique du binôme est ainsi, quoique de manière insensible pour le lecteur, exacerbé par la présence in absentia d'un de ses termes, la mobilité. Ce dispositif sémiotique correspond à la réalité du tableau en trois « volets » qu’est ce roman et que, objectivement immobile, seules les

4. La formule est utilisée par Simon dans son Discours de Stockholm (p. 890).

5. Par exemple, dans Triptyque: «[L']échantillonnage varié des verts [du verger] vu à travers la pellicule sombre est toutefois ramené à une gamme uniforme de verts olive seulement différenciés par leurs valeurs " $(T$, p. 756$)$.

6. On pense ici aux tableaux de Paul Delvaux, Jean Dubuffet et Francis Bacon qui sous-tendent l'écriture (voir A. B. Duncan, "Triptyque. Notice", E I, p. 1420-1432).

7. À l'exception d'une apparition isolée du peu mobile « mobilier ». 
opérations d' " arrangements, permutations, combinaisons ${ }^{8}$ " du commentateur (auteur, puis lecteur) sont à même de mettre en mouvement. Triptyque est bien ce que désigne son titre: non pas la narration d'événements se déroulant dans trois diégèses différentes, mais la mise en relations de trois images immobiles (une carte postale d'une station balnéaire, une affiche représentant une scène urbaine, un puzzle figurant un village et ses environs), qui s'en trouvent chacune animées et diégétisées.

Le narrateur-observateur se montre tout particulièrement captivé par le "va-et-vient ${ }^{9}$ " entre le mouvement des corps et leur inertie, qui d'ailleurs, ainsi que l'a souligné la critique dès la sortie du roman ${ }^{10}$, fonde la cinématographie, qui y occupe une place cruciale et qui est une des deux activités artistiques qui nourrissent le plus Claude Simon, outre la littérature. Quant à l'autre, à savoir la peinture, on sait que la palette de couleurs dont use l'ancien peintre joue un rôle structurant dans son travail d'écriture ${ }^{11}$. Mais on a moins à l'esprit le fait que les qualités chromatiques et les harmonies de cette palette dépendent de l'(in)action des corps peints et donc de leur localisation plus ou moins (in)stable dans l'espace et dans le temps - telle cette mouette posée sur la mer dans Sodome et Gomorrhe et dont la description évolutive par Proust est pour Simon "peut-être le plus grand tour de force que l'on ait jamais réussi en littérature ${ }^{12}$ ".

Tout le potentiel poétique des corps se trouve enfermé dans l'activité à laquelle le corps humain se livre de la façon la plus intense et la plus totale: l'activité sexuelle. Le va-et-vient et, généralement, le contraste entre le repos et l'agitation en sont des aspects constitutifs, le coït débouchant sur l'immobilité la plus totale ${ }^{13}$. En matière sexuelle, l'observation minutieuse est pour ainsi dire à la fête, à l'image des deux jeunes voyeurs dont le plaisir - composante de l'écriture et de la lecture jugée essentielle par Simon - a une fonction de mise en abyme. Presque la moitié des pages (65 sur 142) contient un passage explicitement érotique, sans compter les nombreuses

8. Voir C. Simon, «La fiction mot à mot », E I, p. 1201.

9. Sept occurrences dans le texte, en plus des dizaines de mots (" remous ", "balancement ", " oscillation ", etc.) et formules (" allant et venant », " allées et venues ", "s'étendant, se rétractant », etc.).

10. A. B. Duncan, art. cité, p. 1424.

11. B. Ferrato-Combe, Écrire en peintre. Claude Simon et la peinture, Grenoble, ELLUG, 1998, p. 210-217.

12. J.-C. Lebrun, "Claude Simon: "Parvenir peu à peu à écrire difficilement” ", L'Humanité, 13 mars 1998.

13. Voir par exemple T, p. 781 ou 864. 
autres pages que pareille ambiance érotise à partir de tel ou tel mot ou de telle ou telle situation équivoques.

Dans chacune des principales isotopies narratives, celle du jeune drogué, celle du jeune marié et celle de la (très) jeune noyée, un acte sexuel joue le rôle de catalyseur antinomique, agréable et néfaste à la fois, vivifiant et destructeur. L'observation des corps n'a pourtant rien d'une inquisition, ni d'un voyeurisme: elle procède d'une curiosité certes non dissimulée, mais très soucieuse de neutralité. Mi-impassible, mi-empathique, elle fait preuve d'une fascination maîtrisée, servie par le style tâtonnant de Simon, qui procède par touches et retouches ("non pas..., mais...", "soit que..., soit que...") et est l'équivalent, dans l'ordre de l'écriture, de l'investissement physique du peintre que Simon a été et qu'il est devenu toujours plus littérairement. L'observation est aussi investie qu'animée de neutralité axiologique, de "Wertfreiheit", pour utiliser le mot de Max Weber dans un sens non pas scientifique, mais spécifiquement littéraire ${ }^{14}$.

Afin de mieux cerner cette empathie avec les corps, il convient justement de la considérer comme telle, c'est-à-dire comme une projection dans des corps opérée par une entité écrivante qui, de ce fait, est elle-même dotée de corporéité. Précisons ce point. Quand, à partir du Vent (1957), Simon met au cour de sa conception du roman la notion de mémoire, son projet d'écriture en vient à se désigner comme un investissement du corps écrivant dans ce qu'il écrit, comme une textualisation de ce corps. En se faisant autographique, ce projet déjoue à la fois le piège du faux dilemme entre " réalité " et " fiction ", la vaine tentation autobiographique et la tout aussi vaine occupation autofictionnelle ${ }^{15}$. Il évite aussi l'enfermement dans une conception magique du texte censé s'engendrer tout seul sous l'effet de quelque vertu interne. L'écriture est certes un « extraordinaire processus qui fait que, parti avec un vague projet, on voit peu à peu se faire au fur et à mesure de l'écriture "autre chose" ", explique le romancier en 1972, mais en ajoutant: « non que cela se fasse tout seul, est-il besoin de le préciser $^{16}$ ? "

14. Dans Le Savant et le politique (trad. J. Freund, Plon, 1954), Max Weber désigne ainsi la « neutralité axiologique » du chercheur en sciences sociales.

15. Car, comme le déclare Simon, "à quoi bon inventer quand la réalité dépasse à ce point la fiction?" ("Et à quoi bon inventer? ”, entretien avec Marianne Alphant, Libération, 31 août 1989; repris dans les Cahiers Claude Simon, n 11, 2016, p. 19-24).

16. C. Simon, «Réponses de Claude Simon à quelques questions écrites de Ludovic Janvier ", Entretiens, $\mathrm{n}^{\circ} 31,1972$, p. 29 ; repris dans les Cahiers Claude Simon, n 9, 2014, p. 9-23 et en ligne sur le site de l'Association des Lecteurs de Claude Simon. 
C'est même depuis le tout début de sa trajectoire littéraire que le corps de l'écrivain insiste: dès Le Tricheur, roman déjà travaillé par le "magma " mémoriel, il se montre à l'étroit dans le format hégémonique du roman " réaliste ». Puis, dans $\mathrm{La}$ Corde raide, il s'autorise à délaisser la trame fictionnelle pour un dispositif plus explicitement autographique et réflexif. Il y affirme comme une urgence son statut d'écrivain, pourvu d'un corps attaché par toutes ses fibres à l'indépendance de l'activité littéraire. Évoquant son corps d'écrivain sur " cette sacrée corde raide " qu'est la vie, il s'érige contre «toutes les lois, recettes et dogmes infaillibles pour se bien conduire dans la vie et avancer en équilibre, sans risque d'aucune sorte, dans une attitude gracieuse et virile, les yeux levés fixant le ciel des réalités objectives et le petit doigt sur la couture du pantalon " $(C R$, p. 61). Cela sonne comme un appel à l'insoumission de son corps socialisé, un appel à sa propre émancipation physique, morale, politique et économique.

Appel qui n'est toutefois pas motivé par un projet de changement du monde par la littérature, d'engagement au sens sartrien, mais qui s'avère toujours plus porté par une aspiration à la liberté de l'écrivain en tant qu'écrivain: un appel à l'insoumission de son corps « littérarisé ${ }^{17}$ ", point de départ d'un engagement littéraire ${ }^{18}$. À partir du Vent, Simon invoquera en effet le pouvoir spécifique de la littérature dotée de son propre (autos) principe fondateur $\left(\right.$ nomos $\left.^{19}\right)$, consolidant ainsi son autonomie. Cette consolidation implique chez lui de délester son écriture de tout ce qui lui paraît être contrainte romanesque injustifiée, non pas en tant que contraignante, mais en tant que pseudo-romanesque, car issue de logiques (philosophiques, psychologiques, etc.) non littéraires. Le travail d'écriture de Claude Simon est un combat double, d'une part contre l'hétéronomie, c'est-à-dire l'intrusion de tout principe autre (heteros nomos) que littéraire (l'argent, le pouvoir, etc.), d'autre part contre la tendance du nomos littéraire à figer des procédés inédits en usages sclérosants - et à les figer y compris dans son propre corps d'écrivain. Son œuvre est le lieu d'un effort inlassable visant à réduire le

17. C'est-à-dire socialisé sur un plan littéraire. À propos de la "littérarisation " de Claude Simon, voir P. Dirkx, op. cit., p. 180 et passim.

18. Le Discours de Stockholm a notamment pour objet cette spécification, surtout dans son développement final, où Simon parle d'" un engagement de l'écriture" ( $D S$, p. 902).

19. Soit « ici, on écrit, on est écrivain ". Voir P. Bourdieu, Les règles de l'art. Genèse et structure du champ littéraire, Le Seuil, 1992, p. 298-321. C'est ce nomos littéraire que Simon défend à Stockholm: être " un simple écrivain " (DS, p. 889). 
conflit insoluble entre la tendance à l'autonomie et la tendance à l'hétéronomie qui tiraille tout écrivain, une tentative d'apaisement de son antinomie.

Ce combat va de pair avec une attention croissante portée aux corps. Progressivement, elle concerne moins ce qui arrive aux corps que les rapports entre ceux-ci ou entre deux états d'un même corps, voire d'une même partie du corps ${ }^{20}$. Simon soumet la corporéité à une analyse d'ordre littéraire des tensions antinomiques dont chaque corps semble être l'instrument et l'enjeu. $\mathrm{Au}$ fur et à mesure qu'il abandonne les ficelles de la doxa romanesque pour donner libre cours à la matière autographique, on le voit qui applique cette analyse à un corps qui jusque-là passait inaperçu, toute l'histoire de l'autonomisation de la langue littéraire à partir de 1850, liée à celle du champ littéraire $^{21}$, déconseillant de le mettre en texte: le corps de l'écrivain lui-même. Ainsi qu'il l'explique peu avant la parution de Triptyque, il ne se sent capable d' "écrire qu'à partir de $[s]$ on expérience personnelle du monde ${ }^{22}$ ». Il prend aussi en compte le fait que son corps est sujet à modification, notamment à travers des processus de socialisation, lesquels, pense-t-il, "dictent " ses différentes options d'écriture: "[Celles-ci] me sont dictées par des impératifs qui [...] découlent de la vie que j'ai menée, de la société et du milieu dans lesquels j'ai vécu, des événements historiques auxquels j'ai été mêlé, de même que de ma libido, mon éducation, etc. ${ }^{23}$."

Cette corrélation entre le projet autographique et l'analyse littéraire du corps de l'écrivain semble contredite par Triptyque et par les autres romans de la période "formaliste" (Les Corps conducteurs et Leçon de choses), marqués qu'ils sont par une forte autoréférentialité et par un narrateur désincarné, fantomatique, radicalement coupé de l'auteur. Or il n'en est rien, car cette désincarnation, métaphorique, loin de renvoyer à quelque absence réelle de l'auteur, ne constitue qu'une option littéraire que ce dernier a jugé utile de prendre à ce moment-là, une prise de position liée à sa position d'écrivain dans un champ littéraire très sensible aux mots d'ordre du champ de la critique (universitaire), ou, pour le dire encore autrement, un investissement dénié de son corps d'écrivain dans une œuvre orientée par celui-ci et

20. Par exemple, dans Triptyque, le sexe du chasseur reprenant son "va-et-vient ", " [c]omme doué d'une vie indépendante" $(T$, p. 860).

21. G. Philippe, "Une langue littéraire?", dans G. Philippe et J. Piat (dir.), La Langue littéraire. Une histoire de la prose en France de Gustave Flaubert à Claude Simon, Fayard, 2009, p. 27.

22. C. Simon, «Réponses de Claude Simon à quelques questions écrites de Ludovic Janvier ", éd. cit., p. 22. 23. Ibid., p. 24. 
par les logiques du champ. Ce corps n'arrête d'ailleurs pas, au contraire, de laisser des traces dans le texte. Dans Triptyque, le narrateur-observateur n'a rien de désincarné: investi dans la diégèse, il y occupe un point de vue (" on peut voir ", passim), il est muni d'un " oil " (p. 745) - et même de tout un corps, ce point de vue étant aussi un point d'ouïe (" on peut entendre ", passim), un point d'odorat (" on peut sentir", passim) et de toucher ("l'ongle peut rayer ", p. 746), bref une instance dotée des cinq sens et donc de corporéité. Ce corps se déploie dans le temps et dans l'espace diégétiques ("l'œil d'abord aveugle commence peu à peu à distinguer ", p. 748) et se montre capable d'y intervenir ("Si on trempe sa main dans la fontaine », p. 749).

En 1961, Simon avait déjà expliqué à quel point ses narrateurs étaient incarnés: "je ne peux écrire mes romans qu’en précisant constamment les diverses positions qu'occupent dans l'espace le ou les narrateurs (champ de la vision, distance, mobilité par rapport à la scène décrite ${ }^{24}$ )". À cela s'ajoute que "le travail de rédaction a amené Simon à puiser ${ }^{25}$ " dans sa mémoire à court, moyen ou long terme. La métaphore du puits est d'autant plus pertinente que la mémoire a partie liée avec le réceptacle à partir duquel les schèmes cognitifs et pratiques incorporés par un individu tout au long de sa vie orientent ses attitudes et aptitudes ${ }^{26}$. Dans Triptyque, cette mémoire liée au corps de l'écrivain est tellement envahissante qu'elle vient comme troubler la coulée imperturbable de ce « récit " presque dépourvu d'alinéas. C'est peut-être surtout le cas des pages qui évoquent la recherche nocturne du corps de la fillette disparue et qui s'originent dans un drame familial qui hante la mémoire et l'œuvre de Simon depuis les premières lignes de son premier roman ${ }^{27}$. On y voit le narrateur-observateur projeter sa corporéité en saturant la diégèse de corporalisations: le "chuintement " de la cascade est "[p]uissant, figé et omniprésent "; les "petites lumières qui dansent çà et là vont et viennent ", etc. (p. 872). Cette corporalisation tous azimuts de la nature et des phénomènes matériels, derrière laquelle disparaissent les corps humains à la recherche du corps disparu, va jusqu’à transformer le

24. «Réponse à une enquête ", Premier plan, nº 18 (octobre 1961), cité dans A. B. Duncan, op. cit., p. 1433. 25. A. B. Duncan, op. cit., p. 1421.

26. Voir notamment, C. Simon dans L'Herbe (p. 14). La mémoire participe en effet à cette « connaissance par corps " que Pierre Bourdieu appelle « habitus " (Méditations pascaliennes, Le Seuil, 1997, p. 163).

27. R. Sarkonak, "Comment noyer le poisson, ou le "non-dit" dans Triptyque ", Sofistikê, n 1, 2009, p. 18 (accessible en ligne à l'adresse: [associationclaudesimon.org/IMG/pdf/Sarkonak_Noyer_2009.pdf]). 
monde diégétique en gigantesque corps vivant (" la multiple respiration de la terre nocturne ", ibid.).

Mais c'est l'évocation des corps animés qui finit par trahir le sang-froid du narrateur et, inséparablement, de l'auteur. Car, alors même que les forces naturelles menacent de mort tous les corps et jusqu'à une petite fille innocente, elles vouent ces corps à un destin de copulation, qu'hyperbolise "l'assourdissant grésillement des insectes en rut ", "appelant sans trêve à d'aveugles, impérieux et éphémères accouplements " (ibid.). Fureur universelle qui est redoublée par «la mystérieuse palpitation végétale [...], l'impérieuse et incessante circulation de la sève" (ibid.). En associant aussi étroitement les deux tendances antinomiques qui par excellence se disputent le monde, le narrateur semble suggérer que la mort probable de la disparue est imputable à l'un de ces "aveugles, impérieux et éphémères accouplements " dont il parle. Les dernières lignes de la séquence semblent n'avoir d'autre fonction que de confirmer cette hypothèse: "Par endroits un rameau, une branche courbée sous son poids s'incline jusque dans le courant qui l'entraîne, la relâche, la reprend dans un continu va-et-vient " (ibid.). L'homicide involontaire par excès de zèle amoureux de la part de la servante censée surveiller la petite fille n'aurait pu être mieux suggéré que par cette métaphore érotique (couronnée par le signifiant sexuel par excellence "va-et-vient») aussi bien que létale (la petite mort qui a emporté la servante ayant entraîné, tel le courant de l'eau, la mort de la petite). Ainsi, la corporalisation universelle qu'implique l'érotisation universelle amène le narrateur-observateur au bord de l'émotion, voire d'un sentiment de culpabilité ${ }^{28}$.

L'observation de cette orgie sexuelle cosmique n'en demeure pas moins exempte de tout jugement de valeur moral. D'abord compte « la pertinence des rapports entre [1]es éléments ${ }^{29}$ " de la composition langagière. De nombreuses phrases à caractère sexuel ont une fonction presque ouvertement compositionnelle. Il en va de même pour les multiples évocations, érotiques ou non, de corps coupés, comme le sont certains corps en peinture par l'encadrement du tableau, ou de corps apparemment désarticulés renvoyant à la structure apparemment désarticulée du roman, ou encore de corps " conducteurs » dont tel ou tel geste ménage discrètement une transition vers une nouvelle scène. «La représentation de la sexualité dans Triptyque n'a rien d'une leçon d'érotisme et s'apparente peu à une leçon de transgres-

28. Voir aussi ibid., p. 23.

29. DS, p. 896. 
$\operatorname{sion}^{30}$ ": de même que le roman suivant sera une Leçon de choses avant tout littéraire - «leçon » non pas dans une acception moralisatrice, mais au sens d'incitation à l'expérimentation -, les descriptions érotiques dans Triptyque forment une leçon d'érotisme littéraire et une leçon de transgression littéraire. C'est que la mémoire du corps de l'écrivain est d'abord mobilisée à des fins artistiques et par des stimuli artistiques: tableaux, films, mais aussi lectures accumulées au cours de sa littérarisation.

Pour conclure, on voit que ce dispositif poétique destiné à renverser d'un coup le modèle du roman "à prétention réaliste ${ }^{31}$ ", comme le personnage finit par balayer le puzzle qu'il a patiemment construit ( $T$, p. 883), permet d'ajuster l'écriture, de manière jusque-là inégalée, au corps du scripteur. Rien d'étonnant à ce que le mot " corps " ait, dans ce roman pourtant associé à la mort du sujet, la fréquence la plus élevée de toute l'œuvre ${ }^{32}$.

\section{LE JARDIN DES PLANTES OU LE PASSAGE À L'ACTE AUTOGRAPHIQUE}

Presque un quart de siècle plus tard, Claude Simon reprend l'essentiel des innovations introduites dans ses romans antérieurs, en les dépassant à la faveur d'une nouvelle expérience qu'il intitule Le Jardin des Plantes ${ }^{33}$.

On a vu comment, à force d'observer sur une base toujours plus mémorielle les forces antinomiques et leurs effets sur les corps, Claude Simon en est venu à textualiser de plus en plus son propre corps. Toujours aussi fidèle à ce projet d'autographie que défavorable à l'autobiographie, il entend maintenant donner accès à certains de ses jardins secrets, en les rendant publics pour la cause littéraire (Les Jardins publics était le titre initial du roman). Entreprise réflexive sans précédent, Le Jardin des Plantes met à nu le dédale d'antinomies qui oriente l'écrivain, de même que celui-ci a pu, au gré de ses promenades, saisir et se saisir de la structure labyrinthique du Jardin des Plantes, mélange improbable d'éléments complémentaires et en même temps inconciliables. Il s'y expose donc à travers l'exposé littéraire des antinomies avec lesquelles il est aux prises (notamment sur le plan moral ou dans le domaine de la création artistique) et qu'il livre telles qu'elles,

30. A. B. Duncan, op. cit., p. 1439.

31. C. Simon, «La fiction mot à mot ", E I, p. 1186.

32. Il apparaît presque une fois par page.

33. Le cadre d'analyse du corps de l'écrivain dans Le Jardin des Plantes a été élaboré dans P. Dirkx, «Une politique autonome: l'érotisme littéraire. C. Simon, Le Jardin des Plantes (1997) », dans J. Dubois (dir.), Sexe et pouvoir dans la prose française contemporaine, Liège, PU de Liège, 2015, p. 173-187. 
sans commentaire direct, à travers une écriture au plus près de sa mémoire. Mémoire qui le pousse à mettre en texte non seulement son corps, mais son corps d'écrivain. Car c'est dans Le Jardin des Plantes que Simon apparaît au grand jour en tant qu'écrivain et même en tant qu'auteur de ce livre, comme s'il s'autorisait enfin à laisser monter sur scène celui qu'on a vu progresser dans les coulisses durant plus d'un demi-siècle.

Cette textualisation de l'écrivain en tant que ce qu'il est, un corps écrivant socialisé et notamment littérarisé, s'accompagne de nouveaux progrès sur la voie de l'autonomie scripturale. Le flux mnésique est désormais si peu mis en forme par un sens pratique littéraire ${ }^{34}$ devenu des plus désinhibé qu’il bouscule jusqu'aux règles les plus élémentaires de la langue écrite, donnant droit de cité à d'autres codes et registres de la langue, déroutinisant les usages syntaxiques, typographiques, etc. La langue littéraire, quant à elle, est constamment déstabilisée par la présence de catégories discursives " déplacées » (interview journalistique, archives, actes de colloque, etc.). Si bien que c'est la notion même d'écriture qui se trouve remise en cause, privée de son caractère d'évidence éternelle, rassurante et plaisante qui est un des socles de "l'ordre établi ${ }^{35}$ " littéraire. Et tout cela dans un roman qui, se revendiquant comme tel sur la couverture, ne cesse de malmener les règles les plus communes du genre.

Cette déconstruction est corrélée à la charge réflexive du roman. À mesure que l'autographie devient texte, elle ne cesse de se dévoiler dans sa vérité de produit d'un corps littérarisé devenu corps d'écrivain. Surtout à travers l'entretien avec le journaliste, "S. » ne cesse de se désigner comme Claude Simon, en révélant nombre de principes distinctifs inscrits dans la position qu'il occupe dans le champ littéraire. Parallèlement, le style " explorant ${ }^{36}$ " de Simon s'y montre plus que jamais inquiet de capter les moindres tensions antinomiques qui agitent les corps observés, le sien propre ou d'autres, indifféremment animés et inanimés. D’un point de vue compositionnel, c'est le corps qui donne au roman sa cohérence ou plutôt son incohérence et sa " discohérence ${ }^{37}$ ", c’est lui qui se signale comme le ferment mnésique de

34. Voir P. Bourdieu, Le Sens pratique, Minuit, 1980.

35. DS, p. 889.

36. C. Simon, "La fiction mot à mot ", $E I$, p. 1201.

37. J. Ricardou, Nouveaux problèmes du roman, Le Seuil, 1978, p. 231. Ce concept pertinent est étudié dans I. Yocaris, «La discohérence dans Triptyque et Leçon de choses de Claude Simon », F. Calas (dir.), Cohérence et discours, PU Paris-Sorbonne, 2006, p. 399-408. 
ce formidable bouillonnement discursif qu'est Le Jardin des Plantes, comme nous allons le voir maintenant.

Il est en effet possible, en remontant à sa source corporelle et en échelonnant toutes les isotopies narratives ou plutôt mémoratives qui en découlent, de reconstituer au moins en partie le delta discursif du texte ${ }^{38}$. Celui-ci donne à voir $\mathrm{S}$. nu devant un miroir, en train de raconter, à sa femme prenant un bain, un voyage en URSS dont il vient de rentrer. Les autorités de ce pays ont essayé de lui faire signer un texte officiel, faisant l'éloge d'une nouvelle URSS en pleine "libéralisation» (perestroïka) sous Gorbatchev. Ce dernier a prononcé un discours que $S$. se remémore en ces termes:

son pays ouvert à tout maintenant prolétaires veaux vaches vers de terre cochons ivrognes droit de tout lire tout voir tout entendre livres étrangers revues étrangères journaux étrangers disques films coca-cola et cetera et cetera tout absolument tout Sauf! Il a dit Sauf! Visage tout à coup sévère plus badin du tout plus question humanisme coca-cola Intraitable résolu inflexible: Sauf pornographie! Pornographie streng verboten (JP, p. 912)

Ce discours met de bonne humeur $\mathrm{S}$. et la baigneuse, qui se rapprochent l'un de l'autre et se mettent à faire l'amour. Mise en place dès le début du texte qu'elle surplombe en réapparaissant vers sa fin, cette scène énonciative-mémorative est, on le verra, au principe d'une partie importante de l'arborescence discursive. Elle est un foyer qui érotise l'ensemble du roman (30\% du nombre total de pages contiennent au moins un passage explicite) et qui lui donne une portée politique, le passage à l'acte apparaissant comme une protestation de S. contre l'interdit "Sauf pornographie! » et comme un acte qui, motivant des pans entiers du roman, illustre à quel point il a passé sa vie à se heurter aux injonctions politiques et morales de toutes sortes. Si la "pornographie " est le péché ultime aux yeux du leader soviétique, la pratique érotique est encore la meilleure façon de le récuser et de récuser le projet idéologique dont il est le produit. Mais "pornographie " voulant dire "description (graphein) de la débauche (porneia) ", la riposte la plus efficace pour un écrivain est de donner toute sa place à l'érotisme dans son travail, de décrire le corps-à-corps amoureux avec tous les moyens littéraires nécessaires, quitte à en inventer de déviants au regard de la bienséance doxique, et - ultime "débauche ", qui distingue Le Jardin des Plantes de Triptyque - de faire porter cette description sur son propre corps en pleins ébats.

38. Une telle reconstitution ne nous parait pas incompatible avec le "bricolage " qui, selon le mot de Simon, a présidé à la composition du roman et qui, lui aussi, et peut-être surtout lui, gagne à être rapporté au corps de l'écrivain, dans son principe général comme dans le détail de ses modalités. 
Pour Claude Simon, qui se donne pour unique mot d'ordre la littérature, l'écueil est alors double. Premièrement, il s'agit d'éviter toute écriture pour (pour plus de liberté sexuelle) ou contre (contre la censure politique) : il s'agit d'être écrivain et, partant, d'écrire selon des objectifs littéraires. Le premier de ces objectifs consiste encore et toujours à innover, c'est-à-dire s'interdire de faire un travail qui a déjà été fait, en refusant les automatismes d'écriture néfastes à l'autonomie littéraire. Il n'était probablement guère de défi plus exigeant pour Simon que celui, qu'il avait commencé à relever, on l'a vu, à l'échelle de tout un roman avec Triptyque, d'une écriture du jeu amoureux portée par les seuls intérêts littéraires du scripteur: le défi d'un érotisme littéraire. Mais le problème s'avère maintenant plus ardu : l'auteur du Jardin des Plantes se trouvant placé frontalement face à lui-même, nu devant un miroir, incliné par toute sa trajectoire à écrire non seulement en tant que corps d'écrivain, mais à écrire ce corps d'écrivain, il s'agit, deuxième écueil, d'éviter de se mettre en scène comme on se donne en spectacle, à savoir en cherchant d'abord à plaire au lecteur, voire à l'émoustiller.

Pour ce qui est d'une écriture autonomisée de l'érotisme, Claude Simon déclarait ceci, un an après la sortie de Triptyque:

je traite les sujets sexuels exactement comme je traite tout le reste. Depuis l'instauration du christianisme, tout ce qui concerne la matière et le corps (que le christianisme opposait à l'esprit et à l'âme de même qu'aujourd'hui on oppose la forme au contenu) a donné lieu à une longue série de censures [...]. Alors, je pense que ce ne serait pas mauvais de dépouiller les sujets sexuels de tout cet attirail religieux [...] et de les décrire comme on décrirait un voyage, une bataille, un poisson, ou un brin d'herbe ${ }^{39}$.

Sauf à avoir lu ces lignes, rien ne permet au lecteur de Triptyque de se rendre compte de l'entreprise de résistance littéraire que Simon mène à l'encontre du moralisme chrétien et de sa logique binaire, à laquelle il ne croit pas davantage qu'à l'opposition de "la forme au contenu ». En revanche, dans Le Jardin des Plantes, cet entretien est comme digéré par le roman. Celui-ci comporte même plus d'une réminiscence qui thématise plus ou moins explicitement le dualisme de l'âme et du corps (par exemple p. 1115), y compris relative au corps du Christ mort (p. 951) ou à la consommation eucharistique du sang de ce corps (p. 967-968). La subversion littéraire a une portée politique qui dépasse de loin celle de Triptyque, dans la mesure où le roman donne cette fois à voir le corps de l'écrivain en proie aux censures,

39. C. DuVerlie, "Interview with Claude Simon », Sub-stance 8 (hiver 1974), cité dans A. B. Duncan, art. cité, p. 1438. 
corps « $\left[s^{\prime}\right]$ efforçant dans [s] on mauvais anglais " de s'exprimer (c'est la toute première " phrase » du roman) et surtout de s'exprimer littérairement, et à la fois corps aimant, souffrant, etc. : corps fait d'antinomies. Ce roman expose, dans les deux sens du mot, les moyens littéraires permettant de cerner non seulement une culpabilité vieille de vingt siècles, mais cette culpabilité telle que Claude Simon l'a incorporée depuis sa naissance, notamment au travers de sa littérarisation. C'est là, sur le plan de la littérature, que réside l'essentiel du caractère provocateur de la scène de la salle de bains. Cette provocation, ou plutôt ce défi lancé à lui-même, Simon a pu le relever dans la mesure où il a assumé d'être l'auteur de son texte, d'écrire celui-ci comme extrait de son corps, non pas simple enveloppe charnelle, mais corps - répétons-le - devenu corps d'écrivain.

En ce qui concerne le second obstacle, à savoir une écriture érotique qui fait de l'œil au lecteur, Simon qualifiait la pornographie de " "non-art" abso$1 \mathrm{u}^{40}$ ", notamment parce qu'il y voyait un moyen d'expression absolument hétéronome, complètement soumis aux diktats du marché de la libido, segment du marché tout court et de la « censure démagogique du Profit, c'està-dire de la grande audience ${ }^{41}$ ". Pas plus qu'aucune autre forme d'écriture littéraire, l'écriture de l'amour charnel n'a à faire appel au lecteur.

Revenons alors à la scène érotique de la salle de bains:

j'entrai dans l'eau transparente vert Nil très pâle elle ne m'arrivait qu'à mi-mollet pied coupé ondulant déformé la cheville comme télescopée angle de réfraction dieu botte fécondant tenon rigide Elle s'est penchée je caressais ses cheveux ses épaules Sur la paroi émaillée de la baignoire l'eau clapotis renvoyait un lacis mouvant réseau de marbrures jonquille polygones dansant s'étirant se contractant se défaisant se reformant j'ai perdu l'équilibre titubant cherchant à me raccrocher aux robinets chavirai dans les éclaboussures $(J P, 916)$

La discontinuité des images et des gestes tient aux caprices de la mémoire, qui associe au " vert Nil » de l'eau des souvenirs plus anciens d'un voyage en Égypte. Cette bigarrure ( $c f$. « marbrure ») ne permet pas au lecteur d'identifier (aisément) la scène comme un acte de fellation en bonne et due forme. La fin un peu périlleuse ("j'ai perdu l'équilibre »), loin d'éveiller ses soupçons, entache à peine le caractère placide de la narration. Dans Triptyque, les plaisirs sexuels étaient perçus avec une empathie flegmatique par le narrateur en position de voyeur ou de spectateur. Ici, le « je ", puis " S. ", est à peine

40. A. Poirson et J.-P. Goux, "Un homme traversé par le travail ", La Nouvelle Critique, n 105, juinjuillet 1977, p. 44 (repris dans ce numéro des Cahiers Claude Simon, voir p. 57).

41. C. Simon, "L'inattendu attendu " [1995], dans M. Calle-Gruber (éd.), Les Triptyques de Claude Simon ou l'art du montage, Presses Sorbonne Nouvelle, 2008, p. 21. 
plus investi, plus concentré, les flashes mémoriels continuant d'affluer, dont ce " dieu botte fécondant tenon rigide " qui désigne le dieu de la fécondité Min et apparaît comme un double de S. en action. Rien n'attire l'attention du lecteur sur le " tenon rigide " de $\mathrm{S}$. qui, de son côté, ne se livre à aucune introspection psychologique, ni considération sociologique ou autre. Ne se donne à lire qu'une écriture avec ses fragiles et expérimentales " combinaisons ».

Mais le tableau érotique de la salle de bains ne révèle toute sa fécondité scripturale que si on le replace dans l'ensemble du texte tel que l'auteur le déroule sous nos yeux. Il est suivi de deux colonnes de texte juxtaposées. Dans la citation ci-dessus, l'énoncé "j'ai perdu l'équilibre " est une litote qui signifie quelque chose comme "je me suis oublié » (ayant le "tenon rigide " et la partenaire s'étant "penchée »). La perte inopinée d'équilibre forme la transition avec la scène suivante décrite dans la colonne de droite, où le cavalier S., pris pour cible en pleine Blitzkrieg de mai 1940, vient de se faire projeter dans l'eau d'un ruisseau par son cheval. Et le « narrateur " de préciser: "Tellement occupé à me battre avec cette jument que je ne me souviens pas d'avoir même entendu les explosions: seulement ces boules de coton gris qui ont commencé à s'élever par-ci par-là dans le pré » (JP, p. 916). L'oubli du monde à cause d'un investissement total dans son monde à soi est ainsi ce qui relie les deux séquences sur le plan du signifié, au-delà des signifiants conducteurs. Dès les premières pages du roman, le plaisir érotique est ainsi associé à la peur de la mort ou plutôt à la non-peur, ou plus exactement encore à l'oubli de la peur.

Pareil oubli est ce que n'arrive pas à comprendre le journaliste venu un jour rendre visite à $S$. pour savoir " comment on faisait pour vivre avec la peur » en mai 1940 (p. 1090). Cette conversation de sourds est le deuxième foyer mémoratif du roman, ainsi subordonné à celui de la salle de bains. Elle est la source d'une constellation d'isotopies liées à la Seconde Guerre mondiale et articulées autour de la mort du colonel du régiment dont faisait partie Claude Simon. Cette scène, qui apparaît dans la plupart des romans depuis La Route des Flandres, figure dans pas moins de $22 \%$ du volume textuel du Jardin des Plantes. Ressentie par Simon comme "le seul véritable traumatisme " de sa vie (p. 1064), cette expérience continue de le poursuivre comme la révélation d'une vérité à laquelle il ne parvient toutefois pas à croire: celle de la coexistence insensée en un seul homme de la pulsion de vie et de la pulsion de mort, soit encore la coexistence entre l'oubli de la mort par investissement total dans la vie et l'oubli de la vie par investissement total 
dans la mort. Simon n'a cessé de fouiller cette antinomie qui, à y songer, court également d'un bout à l'autre de Triptyque, à travers l'oubli mortel de la servante qui aimait trop la vie. Mais elle ne s'y dégageait pas encore de l'opposition, essentiellement formelle, entre mobilité et immobilité, dont elle avait les apparences d'une simple variante. En 1997, l'antinomie entre " mort " (55 occurrences) et "vie» (30) a nettement pris le dessus sur celle entre immobilité (48 apparitions du lexème immobil-, soit une toutes les 7,8 pages seulement, contre 2,8 pour Triptyque) et mobilité (13 contre 0 ).

La recherche intriguée de Simon bute inlassablement sur le dernier geste du colonel qui, alors qu'il venait d'essuyer une rafale de mitraillette, avait encore trouvé moyen de brandir son sabre. S. " ne porte pas de jugement de valeur» sur cette conduite d' " automate » (p. 1117 et p. 1108). Il n'empêche que, chez lui, ce n'est pas la pulsion de mort qui l'a emporté, mais la pulsion de vie: "Jamais je n’avais tant désiré vivre, jamais je n’avais regardé avec autant d'avidité, d'émerveillement, le ciel, les nuages, les prés, les haies... » (p. 1122). Jamais non plus les femmes n'avaient été aussi présentes dans le texte, en particulier celles qui jouent un rôle dans son éducation sexuelle.

Ainsi, l'oubli de soi érotique suscité dans la salle de bains par la violence symbolique contenue dans le "Sauf pornographie! » apparaît comme le versant positif en temps de paix de l'oubli de soi mortifère en temps de violence guerrière. Mais l'acte « idiot » du colonel du point de vue de " "l'art de la guerre", comme on dit» (p. 1117), s'oppose surtout à l'art tout court. Celui-ci est au cour de l'autre colonne de texte qui suit l'agitation dans la baignoire. Il s'agit de la description d'un tableau de Gastone Novelli, artiste avant-gardiste italien, qui donne à voir des rangées de seins nus numérotés. Leur pouvoir érotique est contrarié par cette représentation sommaire et par l'absence d'autres parties du corps. Le tableau est intitulé «Archivio per la memoria ": il s'agit pour l'artiste de faire ouvre à partir de sa propre mémoire. Si Simon, rescapé de la campagne de Belgique puis d'un stalag, fait de sa vie la matière première de son travail artistique, Novelli fait de même à partir de son expérience de survivant d'un camp de concentration puis de la jungle amazonienne. Le tableau n'a pas pour référent cette expérience, mais, comme chez Simon, il l'intègre esthétiquement, jusqu'à n'être plus qu'un tableau.

Notons enfin que la portion de texte de droite (S. avec sa jument) a la forme d'un triangle qui s'évase au fil de la lecture, l'autre portion (le tableau de Novelli) faisant un triangle symétrique posé sur son sommet. 
Ces deux triangles sont ainsi séparés par une bande diagonale blanche qu’il serait difficile dans ce contexte de ne pas associer à un phallus - de la même manière que la toile de Novelli laisse deviner en son centre, comme le précise Simon, "la forme d'un phallus monumental en érection» (p. 917). Cette mise en abyme typographique du tableau achève d'attester que Simon reprend à son compte les fondements de l'esthétique de son ami italien. Les deux textes triangulaires renferment chacun l'antinomie entre l'art et la mort, la coexistence inconciliable entre le nomos de la vie, qui fonde l'autonomie artistique et l'innovation ${ }^{42}$, et le nomos de la mort, qui entraîne vers l'hétéronomie, la répétition et l'" arriération ${ }^{43}$ ». Cette antinomie a pour axe central, pour pivot, un phallus en papier, incarnation sensible mais passant inaperçu de l'érotisme littéraire qui, générateur et mortifère à la fois, réunit les deux romans traités ici. À ceci près que Le Jardin des Plantes - roman qui se donne les moyens d'un érotisme littéraire enfin accompli car incarné par l'écrivain, engageant celui-ci - penche davantage en faveur de la vie.

42. C'est ce que Claude Simon désigne dans le domaine littéraire par « ce que j'appellerais la littérature vivante" (DS, p. 889-890).

43. Ibid., p. 889. 